\title{
STUDIES ON THE ANTIGENIC STRUCTURE OF THE EYE USING THE FLUORESCENT ANTIBODY TECHNIQUE*
}

BY

D. ST. C. ROBERTS

\section{From the Nuffield Laboratory of Ophthalmology, University of Oxford}

THE cause of many cases of uveitis is unknown and it therefore seemed useful to examine a technique, little used in ophthalmic pathology, which might help to solve some problems. This method is the antigen-antibody technique of Coons and Kaplan (1950), who coupled fluorescein isocyanate to an antiserum with which they then treated fresh frozen sections of tissue. The site of the antigen-antibody reaction became visible as a green stain when the sections were examined with ultraviolet light. It is thus really a refined histological staining method. It has been used previously on the eye by Clayton (1954) and Clayton and Feldman (1955) to detect antigens of the embryonic lens, and by Witmer (1955) to study the intra-ocular production of antibody.

Hill and Cruickshank (1953) and Cruickshank and Hill (1953) showed that an antiserum to rat glomeruli could be produced in rabbits, and by using the fluorescent antibody technique they showed that this antiserum reacted not only with the glomeruli, but also with basement membranes elsewhere in the body (spleen, lungs, lacrimal glands, blood-vessels, etc.). These workers did not investigate the reaction of their antiglomerular serum with eye tissues, and therefore the present investigations were made, in order to see whether basement membranes in the eye are similar in nature to those found elsewhere and also whether this technique could be successfully applied to the localization of antigen-antibody reactions in the eye.

\section{Methods}

Anti-rat glomerular serum was produced in rabbits. Six white rats were used to supply the kidneys; the glomeruli were separated, using the method of Hill and Cruickshank (1953), and were preserved in merthiolate $1 / 10,000$ and stored at $-10^{\circ} \mathrm{C}$. The whole glomeruli were injected intraperitoneally into two rabbits, each having six doses at weekly intervals. Blood was removed one week later and the serum coupled with fluorescein isocyanate by the method of Coons and Kaplan (1950). It was found that the staining obtained was not satisfactory using whole serum so the globulin was used. This was prepared by precipitating the serum with an equal volume of 50 per cent. ethanol at $-10^{\circ} \mathrm{C}$., centrifuging the deposit at $-5^{\circ} \mathrm{C}$., and dissolving the precipitate in normal saline, so that the original serum was concentrated to about half its original volume. This solution was dialysed in the cold against buffered saline at $\mathrm{pH} 7.0$ to remove ethanol. Nonspecific staining was removed by repeated absorption with heterologous acetonewashed and dried muscle and liver powders in the cold. The globulin was stored

\footnotetext{
* Received for publication January 16, 1957.
} 
at $-10^{\circ} \mathrm{C}$. in merthiolate $1 / 10,000$. Normal rabbit serum was prepared in the same way; it was coupled with fluorescein isocyanate, the globulins being precipitated and absorbed with heterologous tissue as before.

Cutting of Sections.-Satisfactory frozen sections of the rat eye could not be cut on an ordinary freezing microtome as they disintegrated before being placed on a slide. The method of Linderstrøm-Lang and Mogensen (1938) was therefore adapted. Coons, Leduc, and Kaplan (1951) describe a "Cryostat" in which the microtome is maintained below freezing; in the present experiment a commercial deep-freeze cabinet ("Coldrator", $3 \mathrm{cu}$. ft.) was used. This has a freezing compartment measuring $21^{\prime \prime} \times 11^{\prime \prime}$ and $20^{\prime \prime}$ deep with a removable lid. A sledge microtome was used, and a piece of Perspex laid across the top of the cabinet acted as a shield. Gloves were worn when using the microtome to prevent it becoming coated with frost. A thermometer bulb fixed to the microtome block showed that a temperature of $-10^{\circ}$ to $-15^{\circ} \mathrm{C}$. could be maintained under these conditions during cutting, the optimum being around $-10^{\circ} \mathrm{C}$.

White rats of about $60 \mathrm{~g}$. were killed with ether and the eyes and kidneys rapidly frozen in liquid air. In cutting sections the knife window described by Coons, Leduc, and Kaplan (1951) was found essential to prevent curling. Sections of the eye $25-30 \mu$ were the thinnest that could consistently be produced, those of the kidney being about $6 \mu$. Cutting very thin frozen sections of a complete rat eye proved difficult. If such sections are wanted it is probably better to use only small pieces of tissue. Sections were dried under a fan, fixed with 96 per cent. ethanol at $37^{\circ} \mathrm{C}$. for $20 \mathrm{~min}$., and stored at $-10^{\circ} \mathrm{C}$. They were usually used within a few days. Fixation proved necessary to retain the retinal structure after prolonged immersion in solutions of globulin.

Examination of the Sections.-Alternate sections were treated at the same time with fluorescent normal globulin or with fluorescent anti-rat glomerular globulin (ARGG). They were kept moist in a closed container at $37^{\circ} \mathrm{C}$. for $2-3 \mathrm{hrs}$; surplus globulin was then removed by washing in normal saline for $15 \mathrm{~min}$. in a Coplin jar. The sections were dried under a fan and examined immediately; but, if kept at $-10^{\circ} \mathrm{C}$., the specific staining was visible for many weeks. As a further check, absorption experiments were carried out. In these experiments, sections were treated with non-fluorescent normal or ARG globulin for $3 \mathrm{hrs}$ at $37^{\circ} \mathrm{C}$. before being stained with fluorescent globulin, as before. Confirmation of specificity was obtained when, in those sections absorbed with ARGG before being treated with fluorescent ARGG, the staining was either completely prevented or markedly reduced. Absorption with normal serum did not, however, alter the staining obtained with the fluorescent antiserum.

In examining the sections, light of about $360 \mathrm{~m} \mu$ was used, provided by a Mazda ME/D 250-watt mercury arc lamp with a glass envelope, a glass collecting lens, surface-aluminized mirror, and an ordinary microscope with glass substage condenser. Filters in the illuminating system were Chance $\mathrm{OX} 1$ and a $\mathrm{CuSO}_{4}$ tank; on the eyepiece was a pale yellow UV filter. It was found that some mounting media tended to auto-fluoresce; also, fragments of the lens floated about the section; the sections were therefore examined dry without a cover slip. Photomicrography was carried out using Ilford HP3 or PanF 35-mm. film developed in Microphen. An exposure of 2-4 min. was needed. 


\section{Results}

Kidney.-Studies of the kidney were made both to confirm the potency of the antiserum and to compare the efficiency of the present technique with that of Hill and Cruickshank. In all sections stained with fluorescent ARGG, a thin tortuous line was visible in the glomeruli; a fainter line was visible around the tubules (Fig. 1).

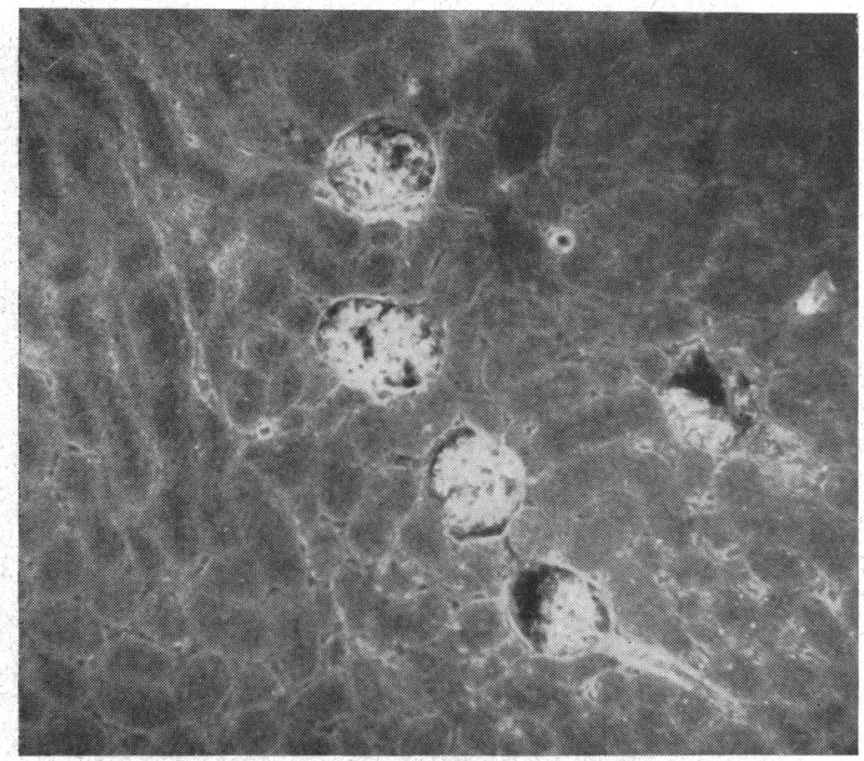

FIG. 1.-Rat kidney (glomeruli and tubules) stained with fluorescent ARGG after section had been treated with normal rabbit globulin. $\times 190$

The larger blood-vessels showed marked auto-fluorescence which masked the specific staining. The picture was in strong contrast to that seen in the controls, which showed only a diffuse non-specific green and in which the glomeruli appeared darker than their surroundings (Fig. 2, opposite).

Specific staining was almost completely prevented by pre-absorption with non-fluorescent ARGG (Fig. 3, opposite).

Eye.-Specific staining with ARGG occurred in the following structures:

Lens.-When the unstained lens was examined with ultraviolet light, the capsule was only faintly visible, whereas the lens substance showed a marked auto-fluorescence. In sections stained with fluorescent normal rabbit globulin, the whole lens including the capsule was faintly green-a nonspecific effect (Fig. 4). When treated with fluorescent ARGG, the capsule alone was heavily stained (Fig. 5). This staining was prevented in other sections (Fig. 6) by treating them with non-fluorescent ARGG before staining with fluorescent ARGG. Specificity of staining was thus confirmed.

Iris and Ciliary Body.-The thickness of the sections prevented a study of fine detail. A definite membrane beneath the ciliary epithelium and within 
FIG. 2.-Rat kidney (glomeruli and tubules) stained with fluorescent normal rabbit globulin. $\times 190$
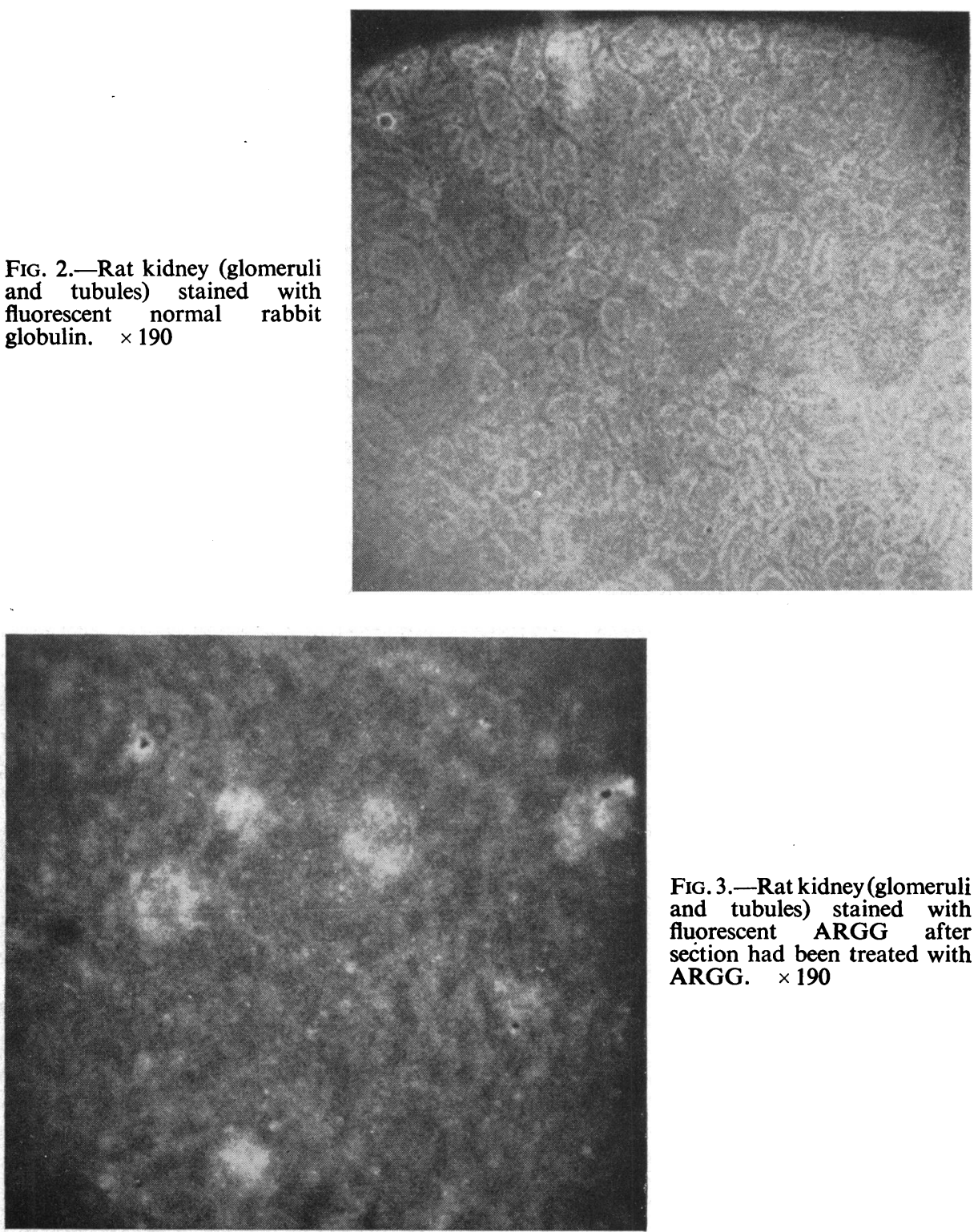

Fig. 3.-Rat kidney (glomeruli and tubules) stained with fluorescent ARGG after section had been treated with ARGG. $\times 190$

the ciliary body was visible. In the iris, a membrane could be seen between the epithelium and stroma, extending into the latter. Staining around the sphincter iridis was much heavier in the ARGG-treated sections than in the controls, suggesting that here too there was a specific antigen. Confirmation was again obtained by absorption (Figs 4, 5, 6, 7, 8, 9, overleaf).

Cornea.-There was so much non-specific staining and auto-fluorescence that any specific staining which may have been present was masked. 

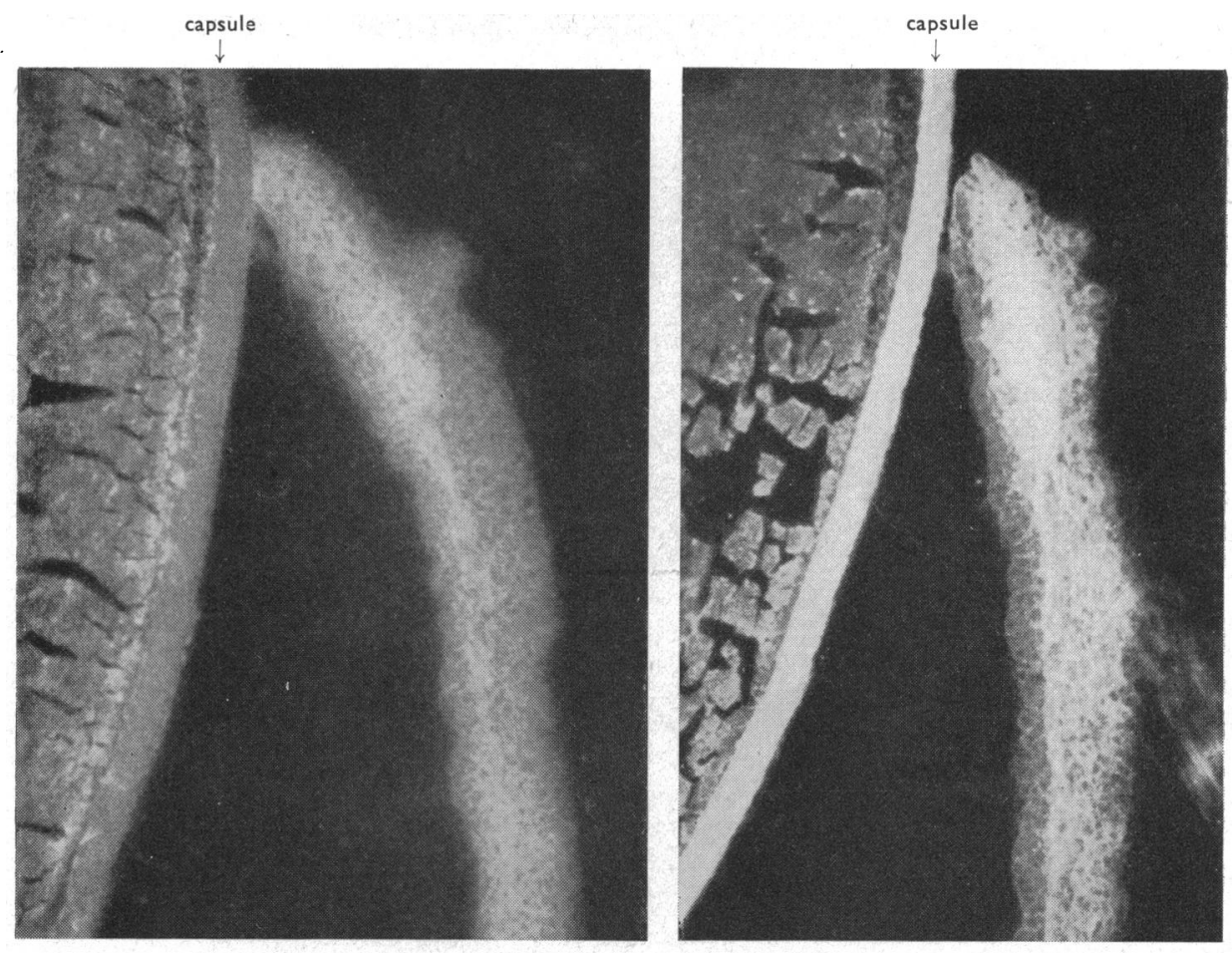

capsule

FIG. 4.-Rat eye (lens and iris) stained with fluorescent normal rabbit globulin. $\times 190$

FIG. 5.-Rat eye (lens and iris) stained with fluorescent ARGG. $\times 190$

FIG. 6.-Rat eye (lens and iris) stained with fluorescent ARGG after section had been treated with ARGG. $\times 190$

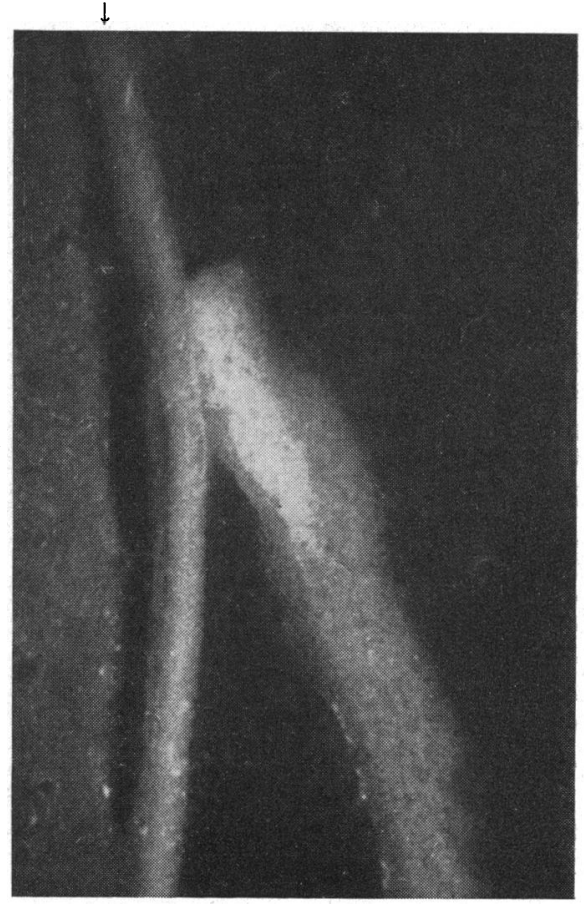




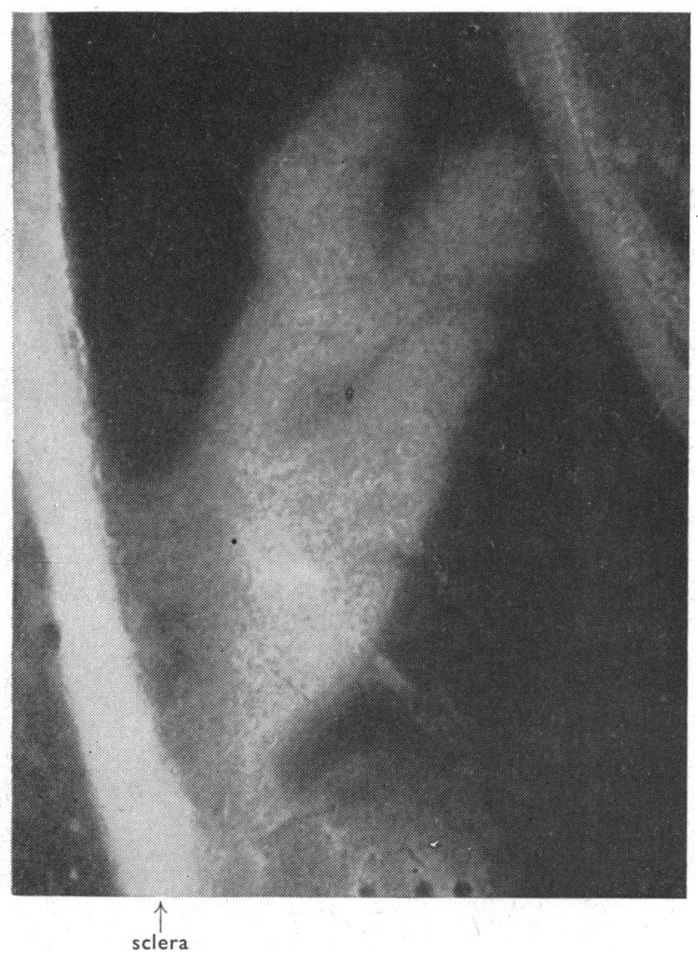

FIG. 7.-Rat eye (ciliary body) stained with fluorescent normal rabbit globulin. $\times 190$

FIG. 8.-Rat eye (ciliary body) stained with fluorescent ARGG. $\times 190$

FIG. 9.-Rat eye (ciliary body) stained with fluorescent ARGG after section had been treated with ARGG. $\times 190$

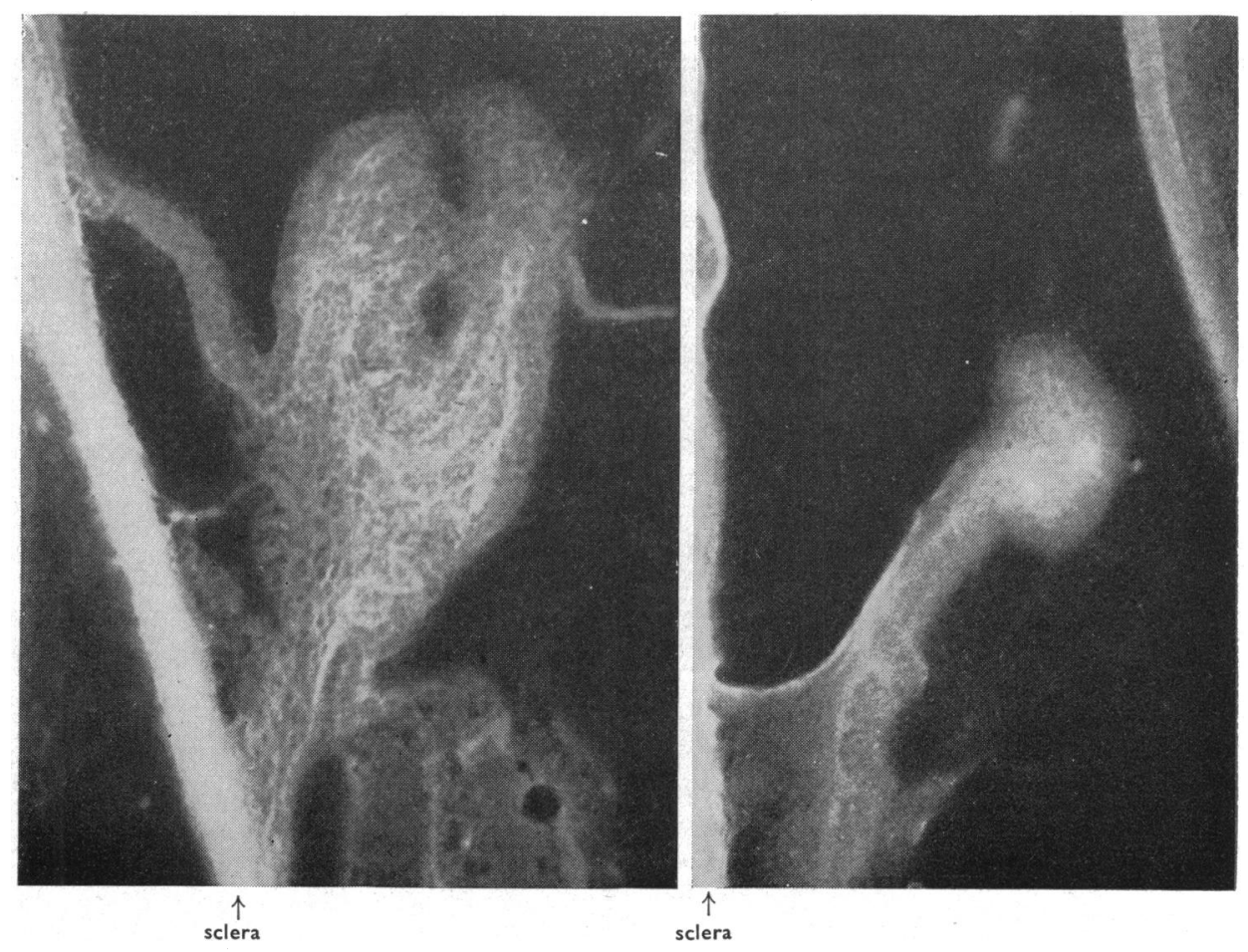


Conjunctiva.-A faint membrane was visible beneath the epithelium. The smaller blood-vessels also showed definite specific staining.

Posterior Segment.-The sclera showed non-specific staining and autofluorescence and again this tended to mask the surrounding structures. The small blood-vessels in both the retina and optic nerve (Figs 10,11, 12) were specifically stained.

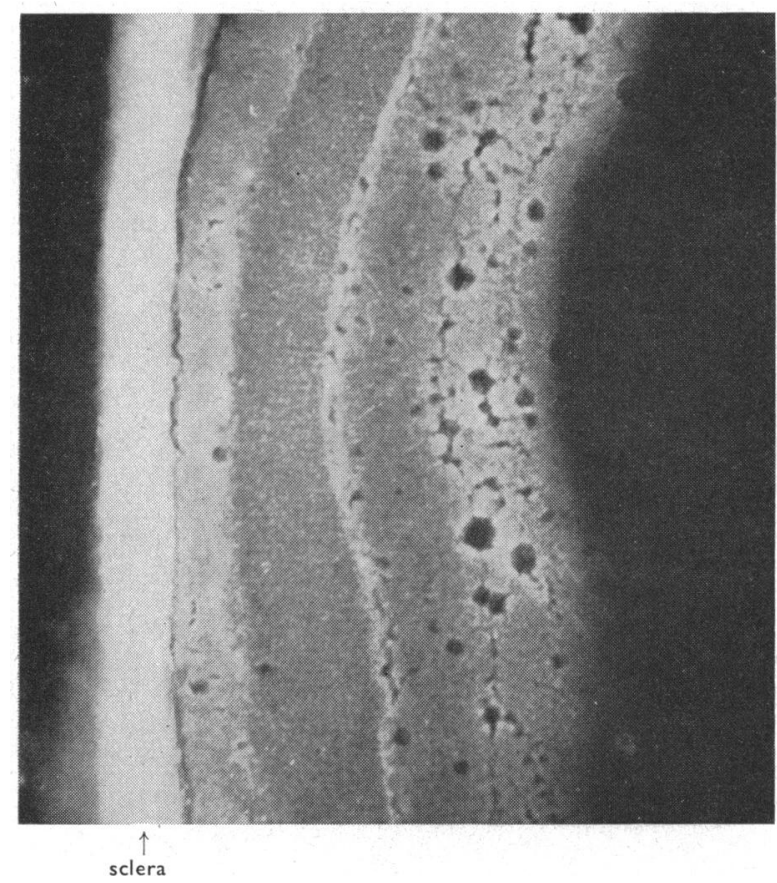

Fig. 10.-Rat eye (retina) stained with fluorescent normal globulin. The holes and cracks are artefacts. The sclera shows auto-fluorescence. $\times 190$

\section{Discussion}

The fluorescent antibody technique of Coons and Kaplan has in the past been used to study a wide variety of problems. These include work on basement membranes by Hill and Cruickshank (1953) and Cruickshank and Hill (1953) and the study of experimental glomerular nephritis by Mellors, Seigel, and Pressman (1955), Mellors, Arias-Stella, Siegel, and Pressman (1955), and Ortega and Mellors (1956). Humphrey (1955) was able to determine the origin of blood platelets and Cruickshank (1956) has attempted to locate the site of formation of anterior pituitary hormones. Witmer (1955) studied the local production of antibody in the eye after an intra-ocular injection of egg albumen.

It has been pointed out in a discussion of the technique (British Medical Journal, 1956) that interpretation of the specificity of staining may be difficult. This is especially so in the eye, where there is auto-fluorescence, particularly in the lens substance and sclera and to a lesser degree in the cornea and retina. 
FIG. 11.-Rat eye (retina) stained with fluorescent ARGG. $\times 190$
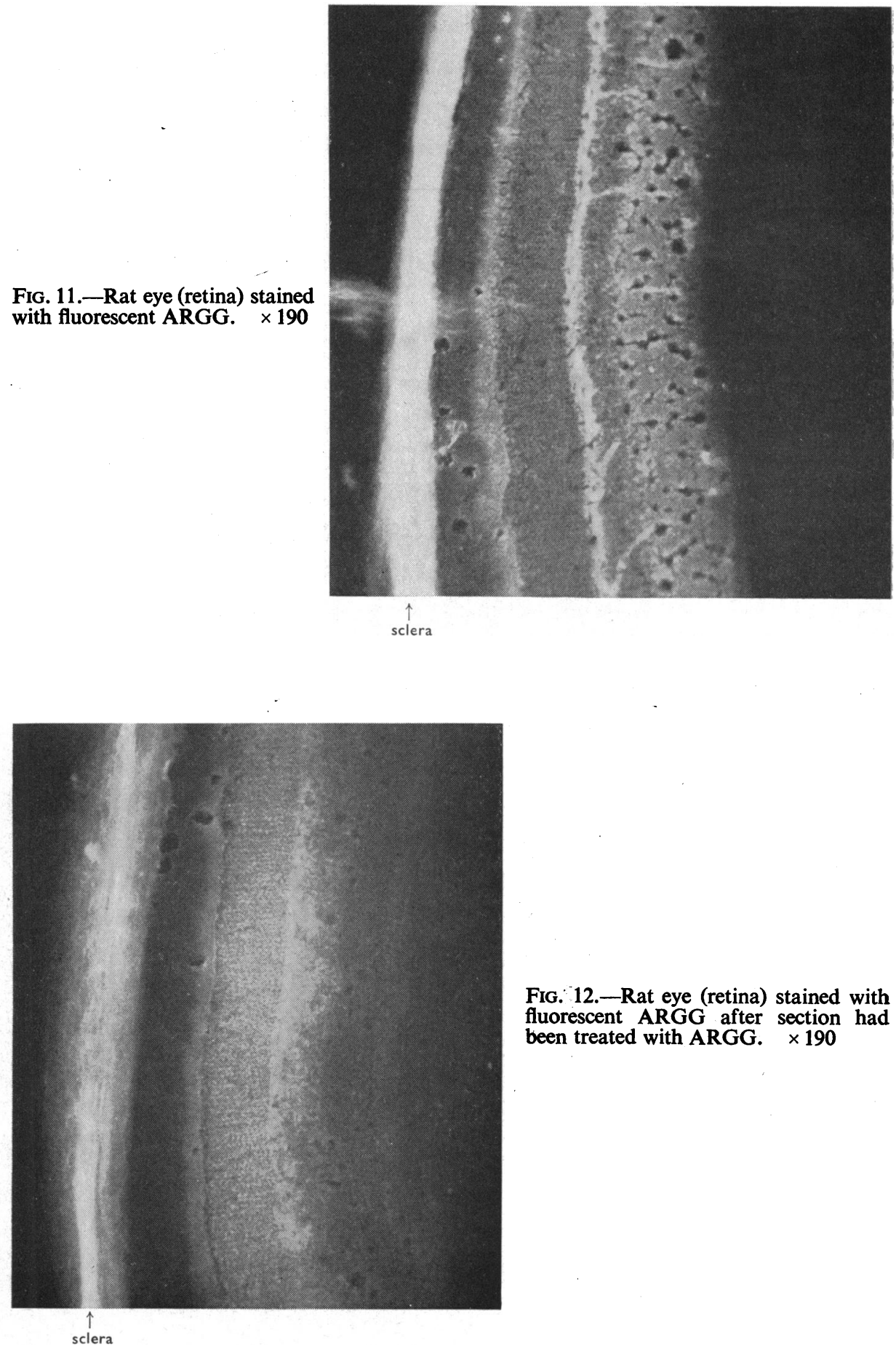

FIG. 12.-Rat eye (retina) stained with fluorescent ARGG after section had been treated with ARGG. $\times 190$ 
Superimposed on this was some non-specific staining, which could not be removed by repeated absorption of the fluorescent globulins with heterologous tissue.

Hill and Cruickshank have shown that antiglomerular globulins react with basement membranes and reticular tissue elsewhere in the body.

In the present experiments, fluorescent ARGG reacted specifically with the glomeruli of rat kidney (Figs 1, 2, 3). In the rat eye, this antiglomerular globulin appeared to be taken up specifically by the lens capsule. It was not possible to differentiate any subdivision of the capsule into zonular lamella and capsule proper. Cruickshank and Hill (1953) pointed out that their serum reacted with reticular tissue but not with collagen. This would support the view that the lens capsule, although showing many similarities with collagen, is in fact different and resembles structureless basement membranes elsewhere in the body (Pirie and van Heyningen, 1956).

In the ciliary body and iris, there was staining of a reticular membrane which appeared to lie between the epithelium and stroma, extending into the latter.

A membrane in the smaller blood-vessels of the retina, optic nerve, and conjunctiva was stained, but in the ciliary body and iris no vessels were demonstrated, the general pattern of the staining had the appearance of connective tissue and could not be identified with any possible blood-aqueous barrier within the anterior uvea (Palm, 1951).

In the albino rats used, Descemet's membrane was difficult to demonstrate even in sections stained with haematoxylin and eosin or with periodic acid Schiff. This difficulty, combined with the presence of non-specific staining and auto-fluorescence, may account for the fact that the membrane was not clearly visible in sections stained with fluorescent ARGG.

The specific staining of these ocular structures would suggest that a common antigen is shared by them and by the glomerular basement membrane.

\section{Summary *}

The application of the fluorescent antibody-antigen technique in ophthalmic pathology is discussed.

An antiserum to rat glomerular basement membrane was produced, and this was shown to react specifically with membranes in the ciliary body and iris and also with membranes in the small blood-vessels of the retina, optic nerve, and conjunctiva.

The lens capsule shared this antigenicity and would seem to resemble basement membranes elsewhere in the body.

I should like to thank Dr. A. G. S. Hill and Dr. D. G. Scott for much advice about the technique, Dr. J. Humphrey for a supply of fluorescein amine, and Mrs. A. Pirie for coupling the sera. 


\section{REFERENCES}

Clayton, R. M. (1954). Nature (Lond.) 174, 1059. and FeldMAN, M. (1955). Experientia (Basel), 11, 29.

Coons, A. H., and Kaplan, M. H. (1950). J. exp. Med., $91,1$. LeDUC, E. H., and KAPLAN, M. H. (1951). Ibid., 93, 173.

CRUICKSHANK, B. (1956). Brit. med. J., 2, 1295. and Hill, A. G. S. (1953). J. Path. Bact., 66, 283.

Discussion ON TECHNIQUe (1956). Brit. med. J., 2, 1295.

Hill, A. G. S., and Cruickshank, B. (1953). Brit. J. exp. Path., 34, 27.

HuMPHREY, J. H. (1955). Nature (Lond.), 176, 38.

Linderstrøm-LANG, K. and Mogensen, K. R. (1938). C. R. Lab. Carlsberg, Série Chim., 23, 27. Mellors, R. C., Siegel, M., and Pressman, D. (1955). Lab. Invest., 4, 69. , Arias-Stella, J., Siegel, M., and Pressman, D. (1955). Amer. J. Path., 31, 687.

OrtegA, L. G., and Mellors, R. C. (1956). J. exp. Med., 104, 151.

Palm, E. (1951). Acta ophthal. (Kbh.), 29, 269.

Pirie, A., and van HeYningen, R. (1956). "Biochemistry of the Eye", p. 30. Blackwell, Oxford.

WITMER, R. H. (1955). A.M.A. Arch. Ophthal., 53, 811. 